Instituto Internacional de Investigación y Desarrollo Tecnológico Educativo INDTEC, C.A.

DOI: https://doi.org/10.29394/Scientific.issn.2542-2987.2019.4.12.14.275-289

OAI-PMH: http://www.indteca.com/ojs/index.php/Revista Scientific/oai

Ensayo Original / Original Essay

\title{
Gerencia Tecnológica de la Información y Comunicación en las Organizaciones Sociales
}

\author{
Autor: Júlmar Eduardo Medina Jiménez \\ Universidad Fermín Toro, UFT \\ meduardo777@gmail.com \\ Guanare, Venezuela \\ https://orcid.org/0000-0003-2504-1784
}

Resumen

El propósito de este artículo en la modalidad de ensayo es repensar la gerencia tecnológica de la información y comunicación en las organizaciones sociales. La metodología para la búsqueda de la información, la cual estuvo sustentada en la revisión documental de algunos planteamientos teóricos de Muñoz y Nevado (2007), relacionados con las organizaciones del siglo XXI, así como de Chiavenato (2006) de la función administrativa que se enfrenta en la búsqueda de definir las acciones tácticas, operativas a través de definición de planes de acción, políticas para potenciar al talento humano como sugiere Mas (2007), de apoyar el papel protagónico de los grupos sociales en la ejecución de los recursos asignados para la solución de las necesidades contextuales de la información, comunicación y conocimientos, donde se despliega como indica la Fundación (COTEC, 1999): una gestión tecnológica asociado a una acción gerencial en las comunidades organizadas en función de la búsqueda de satisfacer necesidades comunes. Se concluyó que en los ámbitos comunitarios debe estar dirigida a la administración de las TIC, como instrumentos confiables para solventar las demandas de la realidad global, que también se manifiesta en los entornos locales, en términos de convocar a la participación y protagonismo para resolver las necesidades colectivas, donde además pueden contar con el apoyo de los entes gubernamentales, a partir de la articulación e integración de los esfuerzos de los ciudadanos.

Palabras clave: gestión; tecnología; tic; organización; sistema social. 


\title{
Technological Management of Information and Communication in Social Organizations
}

\begin{abstract}
The purpose of this article in the essay mode is to rethink the technological management of information and communication in social organizations. The methodology for the search for information, which was based on the documentary review of some theoretical approaches of Muñoz and Nevado (2007), related to the organizations of the 21 st century, as well as Chiavenato (2006) of the administrative function that is faces in the search to define tactical, operational actions through the definition of action plans, policies to empower human talent as suggested by Mas (2007), to support the leading role of social groups in the execution of resources allocated to the solution of the contextual needs of information, communication and knowledge, where it is deployed as indicated by the Foundation (COTEC, 1999): a technological management associated with a management action in the communities organized according to the search to satisfy common needs. It was concluded that in the community areas it should be directed to the administration of ICTs, as reliable instruments to solve the demands of the global reality, which also manifests itself in local environments, in terms of calling for participation and leading role in resolving collective needs, where they can also count on the support of the governmental entities, from the articulation and integration of the efforts of the citizens.
\end{abstract}

Keywords: management; technology; ict; organization; social system.

Date Received: 07-07-2018

Date Acceptance: 03-11-2018 


\section{Introducción}

La gerencia es considerada como un proceso administrativo estructural a partir del cual se despliega una gestión que tiene que ver con las acciones que lideran al talento humano para alcanzar los objetivos corporativos desde una visión global que se manifiesta en los propósitos compartidos por quienes forman parte de ellas, instituyendo los principios básicos que garanticen el administración de las estrategias de acción para la toma de decisiones que se aborden la misión, visión, valores, declaraciones propias de la cultura organizacional en el contexto de las comunidades organizadas.

Cuando una organización genera un plan estratégico, se establecen líneas de acción que permiten llevar a la práctica los procesos participativos de una gestión capaz de congregar los esfuerzos para afrontar los compromisos en términos de asumir los avances tecnocientíficos de una sociedad cada día más globalizada.

De esta manera, la gerencia estratégica está orientada al despliegue de los aspectos tácticos y operativos de una gestión tecnológica congruente con los objetivos de la organización, donde se reconoce la participación desde la gerencia en las comunidades organizadas, basada en el liderazgo del talento humano para posesionarse de las decisiones que correspondan a los requerimientos sociales para determinar la realidad organizacional, de tal manera de poder prever el futuro, que permitan lograr de las metas deseadas en los ámbitos inmediatos de desarrollo sustentable.

El propósito de este ensayo es repensar la gerencia estratégica en la gestión tecnológica de las organizaciones sociales. La metodología para la búsqueda de la información, estuvo apoyada en la revisión documental de algunos planteamientos teóricos relacionados con el contenido temático objeto de esta disertación. 


\section{Análisis de los Fundamentos Teóricos}

Las perspectivas gerenciales en el contexto del siglo XXI, plantean a las organizaciones la adopción de modelos afines a los desafíos de los ambientes globales. Por este motivo, se deben generar una gestión proactiva, dinámica, creativa y adaptada a las innovaciones tecnológicas en la búsqueda de la eficiencia, con el compromiso de alcanzar la calidad de las acciones para hacerle frente a sus necesidades existente en la evolución social de la ciudadanía en diversos aspectos organizacionales de la sociedad.

Las organizaciones, han tenido la necesidad de adecuarse a las situaciones emergentes cada vez más impredecibles, sobre todo cuando el paso del siglo $\mathrm{XX}$ a las nuevas formas organizativas del XXI, las cuales han introducido otras teorías administrativas, técnicas y herramientas gerenciales; según Muñoz y Nevado (2007), los cambios son provocados por:

Las nuevas tecnologías de información que han facilitado las comunicaciones, aumentado la disponibilidad de información, permitido una extensa automatización, haciendo al mismo tiempo que el mercado mundial sean más accesible a un mayor número de empresas. Todo ello ha impulsado la aparición de nuevas formas de trabajo entre las que se destacan: la organización virtual y la estructura en equipos (pág. 45).

Es así, como la gerencia se define dentro de un modelo de organización que Barba (2000), indica es: "consecuencia de la emergencia, maduración y difusión de un nuevo tipo de organización flexible que sustituye al modelo de organización taylorista-burocrático $\mathrm{y}$, al mismo tiempo, ha contribuido al cambio de los paradigmas de las teorías de la administración" (pág. 14). En este contexto, es la encargada de gestionar y potencial al talento humano para responder a las demandas de productividad dentro de un marco global de competencias tecnológicas.

Desde esta perspectivas, los fundamentos teóricos de la gerencia estratégica, buscan establecer la filosofía de gestión para determinar la 
realidad organizacional a partir de la definición de los aspectos que conforman las fortaleza, oportunidades, debilidades, amenazas a las que se enfrenta en la búsqueda de definir las acciones tácticas, así como operativas a través de definición de planes de acción, políticas para potenciar al talento humano como una manera de apoyar a los grupos sociales en el manejo, administración, ejecución de los recursos asignados para la solución de las necesidades contextuales dentro de un marco global de la información, comunicación y conocimientos.

Desde esta consideración, las organizaciones han de hacer énfasis en el desarrollo de talento humano, para que asuma con flexibilidad en su accionar la rapidez vertiginosa de los cambios del entorno tecno-administrativo y social. Al respecto, Sangrá y González (2004), señala que:

Estos cambios no deben analizarse de forma desintegrada y segmentada, sino desde una perspectiva lo más sistémica posible. Así, nos encontramos con que la organización del tiempo en el proceso de aprendizaje podría cambiar de acuerdo con las inmensas posibilidades de comunicación e información (pág. 88).

Si se considera que los escenarios tecnológicos emergentes han impactado la realidad de las organizaciones contemporáneas, por su interdependencia global, signada por la incertidumbre y la complejidad de los cambios generados por las interacciones comunicacionales, el acceso a la información, y la aparición de conocimientos que han llevado a la aparición de nuevos paradigmas gerenciales.

Sin embargo, González (2008a), define la gerencia como un: "macro campo del conocimiento, que tiene como objeto la organización social, para influir en ella y garantizarle, mediante la aplicación de sus conocimientos, una acción competitiva y sobresaliente que le garantice la sobrevivencia en el tiempo" (pág. 47). Esta concepción, representa una alternativa estratégica destinada al desarrollo de las funciones gerenciales como el arte de dirigir las 
organizaciones de manera eficiente y eficaz, asociada con la gestión de los gerentes para alcanzar las metas de productividad en cualquier organización, tanto privada, como pública.

La gestión se sintetiza en el desarrollo de cuatro funciones básicas para coordinar de forma eficaz y eficiente el trabajo de los demás, utilizando para ello las gestiones dirigidas a planear, organizar, dirigir, control, que son propios de la gestión estratégica en la organización. De este modo, el carácter general, flexible y de largo plazo, que se dan a nivel alto e institucional; para desplegar de manera táctica las acciones que se proyectan a mediano plazo en los niveles intermedios y operacionales, donde se reflejan los detalles para alcanzar los objetivos a corto plazo a nivel funcional.

Cabe destacar que la gestión directiva de la gerencia de acuerdo con Chiavenato (2006): concibe a la organización como "la función administrativa que determina por anticipado cuáles son los objetivos que debe alcanzarle y que debe hacerse para conseguirse" (pág. 143); por ellos los gerentes establecen tres niveles de asumen los aspectos (a) estratégicos y abarca toda la organización, (b) tácticos, se dan en los departamentos y unidades, donde está la gerencia media y (c) operacionales, dirigidos a los grupos de personas o tareas.

De ahí que, el incremento de la competencia, el desarrollo acelerado, así como la necesidad de elevar el nivel de productividad en las organizaciones, convoca a una gerencia estratégica, capaz de aprovechar los beneficios de una gestión tecnológica, ligada con los escenarios dinámicos, complejos e inciertos de la realidad de una gestión globalizada, por este motivo, la Fundación COTEC (1999), señala que:

La gestión tecnológica incluye todas aquellas actividades que capacitan a una organización para hacer el mejor uso posible de la ciencia y la tecnología generada, tanto de forma externa, como interna. Este conocimiento conduce hacia una mejora de sus capacidades de innovación, de forma que ayude a 
promocionar la eficiencia y la eficacia para obtener ventajas competitivas (pág. 46).

También es necesario desglosar la gestión tecnológica en los niveles (a) Estratégicos, están dirigidos a establecer los niveles comunicacionales e informativos con toda la organización ya que son de carácter general e institucional; (b) Tácticos, definidos en los procesos técnicos-administrativossociales que se definen en los proyectos a implementar para la transferencia tecnológica; y (c) Operacional, desde la alfabetización, capacitación y donde se reflejan los detalles para alcanzar los planes anteriores, se da a corto plazo y está a nivel operacional.

Razón por la cual, las actuaciones cotidianas de una gestión tecnológica en escenarios de una sociedad del conocimiento que Stehr (2000), explica: está "basada en las estructuras y procesos de la producción material y simbólica, impregnada por operaciones basadas en el tratamiento de la información, el análisis simbólico y los sistemas que dominan la producción de conocimientos" (pág. 63). Por esta razón, abordan la gerencia estratégica en los niveles micro, meso y macro, frente al fenómeno de las comunidades organizadas, debe desplegar una asertividad decisoria que se anticipe a las situaciones problemáticas con sus respectivas soluciones, de las cuales dependerá el éxito o fracaso de la organización.

En tal sentido las Tecnologías de Información y Comunicación (TIC), son consideradas como medios significativos para el desarrollo de cualquier organización, Sugiere Mas (2007): que tienen "el papel protagónico en la sociedad actual, las convierte en un factor fundamental para el mejoramiento de la calidad de vida" (pág. 164). De esta manera las TIC, ha generado un giro en las actividades diarias de las organizaciones sociales, quienes han visto la necesidad de desarrollar sistemas gerenciales abiertos, inteligentes, proactivos, dinámicos, creativos y descentralizados, para adaptarse a un 
mundo cada día más globalizado, ser más competitivas y lograr el éxito.

De esta manera en la gerencia estratégica representa una mirada que conlleva una visión de futuro, a través de gestión tecnológica conscientemente que de manera anticipada, asegura satisfacer los requerimientos de la sociedad globalizada. Según Mintzberg (1998): es relevante asegurar "la integración y complementariedad de sus distintas acepciones: como plan, como pauta, como táctica, como posición y como perspectiva" (pág. 12). Todo esto implica, que se lleve a cabo la meta planeada en la organización en beneficio colectivo de toda la comunidad organizada.

Cuando se habla de comunidades organizadas, se hace inferencia a un aglomerado de personas que usan las redes para satisfacer necesidades comunes de intercambio de información, para lo cual requieren integrar las potencialidades y saberes adquiridos por sus experiencias en los espacios ciudadanos de participación.

La Ley Orgánica de los Consejos Comunales (2009): define a las comunidades organizadas como estructuras como "instancias de participación, articulación e integración entre las diversas organizaciones comunitarias, grupos sociales y los ciudadanos, que permiten al pueblo organizado ejercer directamente la gestión de las políticas públicas y proyectos destinados a satisfacer sus necesidades" (pág. 12). Estas entidades de la tienen amplios niveles de movilización que se han caracterizado por el poder que tienen para tomar las decisiones que les competen con el uso y aprovechamiento de los medios tecnológicos en los ámbitos locales y regionales.

La reapropiación según Rodríguez (2006): tiene varios usos, entre ellos, "la disponibilidad y uso de información para ampliar y diversificar las oportunidades de desarrollo humano. Este papel, desde la perspectiva pragmática, ha contribuido a la apropiación social de las TIC" (pág. 43). Se trata de seguir aprovechando las oportunidades de las interacciones virtuales 
para la concreción de los proyectos locales.

Así, la conformación de las comunidades organizadas en Venezuela, ha visto la necesidad de organizar los aspectos relacionados con visión prospectiva de la gerencia estratégica, capaz de reflexionar sobre las fortalezas, así como de las amenazas que afronta cada entorno social y desde este conocimiento puede aprovechar la gestión tecnológica para llevar a cabo los procedimientos administrativos, técnicos y sociales en el marco de la participación protagónica en la cual se atienda los requerimientos para conseguir optimizar los cánones de vida de los ciudadanos en su incursión a la sociedad globalizada.

La gerencia de la gestión tecnológica desde la perspectiva de Chiavenato (2007a): está destinada a "aplicar una determinada estrategia para alcanzar los objetivos propuestos" (pág. 367). Es decir, es un componente orientador de las acciones que permiten a las comunidades organizadas desplegar un proceso de planificación dinámica, orientada a la consecución de los metas organizacionales de la sociedad, a través del seguimiento de un conjunto de metodologías más efectiva posible para alcanzar la situación deseada u óptima.

Las comunidades organizadas son consideradas instancias de participación, ensamblaje y combinación de esfuerzos de los ciudadanos, estás despliegan una gestión estratégica de autogestión, pero también de cogestión con otras estructuras, entre ellas las de la administración pública, para ejercer directamente la gestión tecnológica a través de propuestas encaminadas a revelar las aspiraciones o requerimientos de los grupos sociales en la construcción de respuestas inmediatas.

Es de hacer notar que las gestiones tecnológicas en las organizaciones sociales, por lo general se desarrollan de manera empírica, inadecuadas para incursionar en una sociedad globalizada que tiene a su disposición herramientas administrativas, tecnológicas y gerenciales, importantes en la 
presentación de cuentas a los órganos competentes, evitando la pérdida de oportunidades en la obtención de recursos para nuevos proyectos en sus comunidades.

La gerencia en la gestión tecnológica, señala Fernández (1993): mejora "la capacidad de interacción y comunicación de los elementos claves del proceso innovador" (pág. 49). Se trata de un conjunto de las políticas públicas, dirigidas al bienestar de los ciudadanos, sometidas a un proceso de transformación enfocado al cambio estructural orientado a desarrollar nuevas y mejores gestiones que tengan en el cumplimiento y desarrollo económico del país.

La gerencia tecnológica, viene a consolidar el fortalecimiento de estructuras comunidades organizadas de acuerdo con Zapata, Murillo y Martínez (2006): permite asumir el "problema de las formas gerenciales y organizacionales, y las estrategias de desarrollo social que se enfrentan a situaciones globales" (pág. 119). Cabe destacar una gestión tecnoadministrativa-social, dirigida al desarrollo organizacional en las comunidades organizadas que buscan tener en cuenta los requerimientos, anhelos y perspectivas de los integrantes en la sociedad; asumiendo las responsabilidades de un desarrollo organizacional para el fortalecimiento de estas estructuras socio-administrativas.

De igual manera las políticas públicas y las necesidades sentidas de las comunidades organizadas, no serán efectivas, si no se implementa una concientización sobre su corresponsabilidad en los valores, actitudes y la búsqueda de la satisfacción por medio de normas y procedimientos técnicos relacionados con las gestiones tecno administrativas de comunidades organizadas en correspondencia con las atribuciones que le confiere la ley.

Al respecto, Chiavenato (2007b): señala que "la administración de las personas se denomina relaciones industriales. Todo estaba al servicio de la tecnología (...) las estructuras organizacionales, se volvieron inflexible y lento 
para estar a tono con los cambios que ocurrirían en el ambiente" (pág. 11). Así, la conformación de una gerencia estratégica, se puede considerar un proceso dinámico, predestinado a cambiar los comportamientos organizacionales en la estructura de participación ciudadana, de modo que esta pueda acoplarse mejor a las nuevas articulaciones sociales, donde administrar, significa hacer las cosas de manera eficiente utilizando las personas y los recursos con que se cuenta para tal fin.

Una gerencia tecnológica en las comunidades organizadas, representa una estrategia dirigida a la participación del ciudadano expresa González (2008b), se despliega en tres niveles "políticos-territoriales de programas, servicios y recursos financieros y no financieros (...) El establecimiento de convenios de descentralización y transferencia entre el Estado y las comunidades supone que cada una de las partes mantiene su autonomía de gestión" (pág. 3). En Venezuela, la asignación y utilización de recursos, de naturaleza financiera o no, depende de las asignaciones presupuestarias a través partidas sustentadas en proyectos de inversión comunitaria, que son expuestas ante las autoridades competentes.

De este modo las comunidades organizadas, asumen proyectos estratégicos de gestión tecnológica de información y comunicación, dirigidas a conseguir un desarrollo local planificado con los requerimientos de los ciudadanos, al punto de ser llamadas "organizaciones inteligentes", las cuales son capaces de evolucionar al ritmo de los requerimientos de la sociedad.

Los señalamientos de Senge (2006): resaltan que un ámbito, donde "la razón de mayor peso para construir organizaciones inteligentes, fue como avances a tientas, hasta que se llegaron a conocer las aptitudes, conocimientos y caminos para el desarrollo" (pág. 13). De Modo que las la gente, es capaz de apropiarse de los beneficios del gobierno electrónico, generador de intercambio de información y comunicación útiles para atender los requerimientos de una dinámica social cambiante propios de las 
organizaciones públicas que impulsen el desarrollo sostenible con miras a fortalecer de forma significativa la gestión tecnológica.

\section{Conclusiones}

A partir de las revisiones de los fundamentos teóricos, se descubrió desde una perspectiva reflexiva que las organizaciones sociales están conectadas indiscutiblemente con los progresos tecnológicos; a través de una gerencia estratégica relacionada con los proyectos sociales encaminados a provocar cambios significativos en el entorno social de participación de los ciudadanos.

Las organizaciones sociales, deben aprovechas las ventajas competitivas de las TIC para el despliegue de las gestiones gerenciales de planificar, organizar, dirigir y controlar las propuestas dirigidas a dar respuesta a los requerimientos de información y comunicación, destinados a proporcionar las interrelaciones entre los sistemas técnicos y económicos del gobierno electrónico atendiendo la complejidad del entono social actual.

Por consiguiente, una gerencia tecnológica, representa una visión estratégica que debe satisfacer los niveles tácticos funcionales a través del uso de las técnicas, herramientas y competencias digitales adecuadas para responder adecuadamente por la gestión tecnológica. El análisis de las situaciones comunes, debe llevar a las decisiones acerca de las acciones estratégicas o tácticas capaces de convertir en operativas las intervenciones en las comunidades organizadas como instancias que muestran sus potencialidades pertinentes en la agilización de las transferencias de recursos técnicos, materiales y financieros para que los proyectos presentados puedan ser ejecutados según lo planificado.

En términos de las organizaciones sociales, implica un ámbito donde la gente desarrolla las aptitudes tecnológicas para crear los resultados deseados, en términos de promover organizaciones inteligentes que aprenden desde sus 
propias experiencias, tanto en la cotidianidad de la convivencia, como en las interacciones virtuales, que utiliza para obtener la información y la comunicación requerida para satisfacer sus necesidades.

Se concluyó que gerencia tecnológica de la información y comunicación, debe estar dirigida al manejo estratégico de las TIC, procurando apropiarse de los beneficios del gobierno electrónico en un cruce de información y comunicación, como resultante de los procesos de aprender a aprender, en términos de atender las demandas tecnológicas de las organizaciones públicas, destinadas a proveer se servicios de calidad dentro de un compromiso comunitario satisfactorios a las demandas de los ciudadanos.

\section{Referencias}

Barba, A. (2000). Cambio Organizacional y Cambio en los Paradigmas de la Administración. Iztapalapa, 48, 11-34, ISSN: 0185-4259. Recuperado de:

http://148.206.53.234/revistasuam/iztapalapa/viewarticle.php?id=659\&l ayout=abstract

Chiavenato, I. (2006). Introducción a la Teoría General de la Administración. Colombia: McGraw-Hill.

Chiavenato, I. (2007a,b). Administración de Recursos Humanos. México: McGraw-Hill.

Fernández, I. (1993). Gestión Tecnológica Competitividad y Empleo. Memorias VI Seminario Latinoamericano. Bogotá, Colombia: Altec.

Fundación COTEC (1999). Pautas Metodológicas en Gestión de la Tecnología y de la Innovación para Empresas. Tomo I: Perspectiva Empresarial. Madrid, España: Gráficas Arias Montano, S.A.

González, S. (2008a,b). Las Organizaciones Sociales y la Gerencia del Nuevo Tiempo. San Cristóbal, Venezuela: Fondo editorial UNET. 
Ley Orgánica de los Consejos Comunales (2009). Gaceta Oficial № 39.335, del 28 de diciembre. Caracas, Venezuela: La Asamblea Nacional de la República Bolivariana de Venezuela.

Mas, M. (2007). Desarrollo Tecnoendógeno. Ensayos sobre Tecnología y Desarrollo Endógeno. Caracas, Venezuela: Editorial Panapo de Venezuela, C.A.

Mintzberg, H. (1998). La Estructuración de las Organizaciones. Barcelona, España: Ariel, S.A.

Muñoz, R., \& Nevado, D. (2007). El Desarrollo de las Organizaciones del siglo XXI. Ética, Responsabilidad Social, Gestión de la Diversidad, y Gestión del Cambio. Madrid, España: Especial Directivos.

Rodríguez, A. (2006). La Brecha Digital y sus Determinantes. México: Centro Universitario de Investigaciones Bibliotecológicas de la UNAM.

Sangrá, A., \& González, M. (2004). La Transformación de las Universidades a través de las TIC: Discursos y Prácticas. Colección Educación y Sociedad Red. Barcelona, España: Editorial UOC.

Senge, P. (2006). La Quinta Disciplina. El Arte y la Práctica de la Organización Abierta al Aprendizaje. Argentina: Editorial Granica.

Stehr, N. (2000). Die Zerbrechlichkeit Moderner Gesellschaften, ISBN: 3934730-18-3. Weilerswist: Velbrück München.

Zapata, D., Murillo, G., \& Martínez, J. (2006). Organización y Management. Naturaleza, Objeto, Método, Investigación y Enseñanza. Colombia: Programa Editorial de la Universidad del Valle. 


\section{Júlmar Eduardo Medina Jiménez}

e-mail: meduardo777@gmail.com

Nacido en Guanare, Venezuela. Técnico Superior en Análisis de Sistemas (IUTEPI, 2001); Licenciado en Administración (UNESR, 2008); Especialista en Gerencia Social (UNEFA, 2011); Administrador del Registro Público del Municipio Ospino, estado Portuguesa. 